\title{
Effects of supplemental measles immunization on cases of measles admitted at the Wesley guild hospital, Ilesa, Nigeria
}

\author{
Kuti Bankole Peter ${ }^{1 *}$, Adegoke Samuel Ademola ${ }^{1}$, Oyelami Akibu Oyeku ${ }^{1}$
}

1. Department of Paediatrics and Child Health, Obafemi Awolowo University, Ile-Ife, Nigeria.

\begin{abstract}
Background: Measles is a highly contagious vaccine-preventable infection which continues to be a significant cause of childhood morbidity and mortality in developing countries particularly those with poor routine immunisation coverage. Supplemental immunisation activities (SIAs) were thus introduced to improve vaccine coverage.

Objective: This study was carried out to assess the impact of the supplemental measles vaccinations on the cases of measles admitted at a tertiary health facility in South west Nigeria.

Methods: Weretrospectivelylooked at therecords of cases of measles in children admitted to the Wesley Guild Hospital, Ilesa over a ten year period (2001 - 2010); five years before and five years after the nationwide commencement of supplemental measles immunisation activities (SIAs) in the region in 2006. Measles cases were defined using the WHO case definition.

Results: Over the ten year study period, a total of 12,139 children were admitted andmanaged; out of which $302(2.5 \%)$ were cases of complicated measles. There was no difference in the mean (SD) of children admitted in the years before and after the introduction of the SIAs $\{6040$ (122.7) vs.6099 (120.2); t-test 0.02, p $=0.988$. $\}$ There was however a remarkable reduction in the proportion of the cases of measles admitted after the introduction of SIAs compared to the period before SIAs $(4.3 \%$ vs. $0.6 \% \times 2=169.580 ; \mathrm{p}<0.001)$

Conclusion:SIAs have remarkably reduced morbidity and mortality associated with measles in the region. We advocate for sustenance of these efforts as well as improvement in routine immunisation coverage to avoid a backlash which can lead to devastating measles outbreak.
\end{abstract}

Keywords: Measles, Supplemental immunisation, Morbidity.

African Health Sciences 2014;14(1): 131-135 http://dx.doi.org/10.4314/ahs.v14i1.20

\section{Introduction}

Measles is a highly contagious, serious childhood exanthematous vaccine-preventable disease. ${ }^{1}$ It is caused by Measles virus - a single stranded RNA

paramyxovirus of the genus morbilliviridae. ${ }^{1}$ It is acquired via droplets or direct contact with nasal or throat secretions of infected persons and occasionally by airborne spread. ${ }^{1}$ Measles has high infectivity rate of more than $90 \%$ which peaks $2-4$ days prior and $2-5$ days following the outset of rash. ${ }^{1}$ Incubation period of the infection may range from $6-19$ days. ${ }^{1-2}$

Measles manifest with prodromal symptoms of fever, cough, conjunctivitis and coryza; then the erythematous, maculopapular rash appear with erosion of the mucosa and pathognomonic Koplik spots. ${ }^{1-2}$ Most deaths following measles often result from viral

\section{*Correspondent author:}

Kuti, Bankole Peter

Department of Paediatrics and Child Health Obafemi Awolowo University, Ile-Ife, Nigeria.

Telephone number: +2348034659848

E-mail Address: kutitherapy@yahoo.com or superimposed bacterial pneumonia, croup and diarrhoea, and are frequently seen in undernourished children. ${ }^{2}$ In addition, measles may be associated with long-term complications including blindness, deafness, chronic lung disease, poor growth, recurrent infections and progressive panencephalitis. ${ }^{2}$

According to the World Health Organization (WHO), measles is a leading cause of vaccine-preventable childhood mortality. ${ }^{3}$ The global fatality rate has however been significantly reduced by vaccination campaigns led by partners in the Measles Initiative: the American Red Cross, the United States Centers for Disease Control and Prevention (CDC), the United Nations Foundation, UNICEF and the WHO. ${ }^{3}$ Globally, childhood mortality due to measles fell by $60 \%$ from an estimated 873,000 deaths in 1999 to 345,000 in $2005 .^{3-4}$ Estimates for 2008 indicate deaths fell further to $164,000 .^{5}$

Despite the availability of safe, effective and cheap vaccine estimated to cost less than one US Dollar to vaccinate a child against measles, the infection still remains a leading cause of vaccine-preventable death among young children in countries with poor immunization coverage and poor health indices. ${ }^{2-3}$ In Nigeria for instance, the 
national routine immunization survey conducted in 2003 revealed that only $12.7 \%$ of children between ages 12-23 months were fully immunized. ${ }^{4}$ Subsequent reports show that immunization coverage in Nigeria over the last decade (2000 to 2010) ranged from 12.7 to $41.0 \%{ }^{5-7}$ Nigeria is ranked in the $188^{\text {th }}$ position among nation of the world with worse immunization records, ${ }^{7}$ hence the introduction of the supplementary measles immunization campaign to increase coverage and reduce morbidity and mortality associated with the infection while making efforts to increase routine immunization coverage.

The primary purpose of Supplemental Immunization Activities (SIAs) is to reach children who have been missed by routine services. ${ }^{3}$ SIAs entail an initial nationwide catch-up aimed at vaccinating all children aged 9 months to 14 years to eliminate susceptibility to measles in the general population. Then there is the periodic follow-up SIAs conducted nationwide every 2-4 years targeting all children aged 9-59 months born since the last SIA with the aim of eliminating any measles susceptibility that has developed in recent birth cohorts. $^{3,13}$

The Federal government of Nigeria in partnership with WHO/UNICEF global immunization vision and strategy, CDC etc launched a two phase national measles supplemental campaign in 2005. ${ }^{6}$ In the northern states of Nigeria where the first phase of the campaign took place in December 2005, about 30 million children were vaccinated. The second phase which occurred in October 2006 captured over 29 million children who received measles vaccine in the Southern states of the country. Follow up SIAs for children 9 -59 months were done every 2 years both the northern and southern states. ${ }^{6}$ This is in addition to the routine measles immunization given as a single dose at nine months in Nigeria.

Evaluation of the impacts of programmes like this is worthwhile to have documented effects of the SIAs on childhood morbidity due to measles. This may serve as encouragement and motivation for increased governmental and non-governmental spending on this cause. This study was carried out therefore to assess the impact of the supplemental measles vaccinations on the cases of measles admitted at the Wesley Guild Hospital, Ilesa, Nigeria.

\section{Methods}

Study design: This is a retrospective observational study.
Study location: This study was conducted at the Paediatric wards of the Wesley Guild Hospital (WGH), Ilesa. Wesley Guild Hospital is one of the tertiary units of the Obafemi Awolowo University Teaching Hospitals Complex (OAUTHC), Ile-Ife, Nigeria. It serves the health needs of the urban and rural communities of the Osun, Ondo and Ekiti states of South west Nigeria. It is a major referral health facility providing both general and specialist paediatric care for these communities. The paediatric department of the hospital admits about 1200 children per annum.

The hospital has four paediatric wards with total beds / cots of 80; thirty-two (32) of which are for newborns. Children with measles and other contagious/infectious illnesses are managed at the isolation children ward with eight cubicles each with 4 beds/cots.

Case notes of children managed for measles are kept in the unit's medical record department and are filed using the International Classifications of Diseases (ICD) coding system to facilitate files retrieval when the need arises. The suspected cases of measles in the unit are reported to the local health authority disease surveillance unit from where the information is relayed to the state and subsequently to national and global records.

\section{Methods}

We looked at the record of cases of measles admitted between year 2001 and 2010 by retrieving the files of all cases of complicated measles admitted during the period. All admitted children less than 15 years during the study period was also noted. The cases of measles were diagnosed clinically based on the WHO case definition criteria thus: ${ }^{2,8}$

a generalized, reddish (erythematous), blotchy (maculopapular) rash with characteristic cephalo-caudal distribution;

a history of fever usually above $38^{\circ} \mathrm{C}$; and

at least one of the following - cough, runny nose (Coryza), or red eyes (conjunctivitis).

Only admitted (complicated) cases were considered for this study.

Children admitted with drug-induced skin eruptions; other exanthematous childhood illnesses not in conformity with the above criteria were excluded.

In addition to the above information, socio-demographic characteristics of the patients were also obtained including age, sex, parental occupation and highest level of education from which social class of the 
parents were obtained using the method described by Oyedeji. ' The proportion of the cases of children admitted for complicated measles during the study period were compared to those admitted during the same period with other diagnoses. Measles cases were managed using standard protocol. ${ }^{2}$

Ethical clearance for the study was obtained from the hospital Physician -in -charge. Informed consent was obtained from the parents/givers of the study participants at admission which was extended to include other studies which may later be carried out using the information recorded in the case note. All information was kept confidential.

Data analysis: The mean (SD) admissions before and after the SIA was compared using students'T - test, while the proportions of admitted measles cases was compared with the proportion of admitted non-measles cases using chi-squared. Statistical significance was fixed at $\mathrm{p}<0.05$.

\section{Results}

Over the ten year study period, a total of 12,139 children were admitted and managed in the Paediatric wards of the WGH Ilesa; out of which 302 (2.5\%) were cases of complicated measles.

\section{Age and gender distribution:}

The ages of the children admitted with complicated measles ranged from 5 to 96 months with a mean (SD) age of 22.2 (18.1) months; and median age of 16.0 months. Sixty three $(20.9 \%)$ of the children were infants. There was male preponderance among the children admitted with measles with male to female ratio of 1.8:1 (194:108). Majority (92.0\%) of the children admitted with measles were from low socioeconomic class.

About half of the total patients were admitted in the period prior to the commencement of SIAs in South Western Nigeria (2001 - 2005) Table 1. No statistically significant difference in the mean (SD) admission before 6040 (122.7), and after the SIAs 6099 (120.2). T-test $0.02, \mathrm{p}=0.988$. There is however remarkable reduction in the proportion of the cases of measles admitted after the introduction of SIAs compared to the period before SIAs (4.3\% vs. 0.6\%) Chi squared 169.580; p < 0.001. (Tables I and II; Figure one).

Table I: Measles cases and total admissions between years 2001 to 2010

$\begin{array}{cccc}\text { Year } & \text { Total admissions } & \text { Measles cases } & \text { Percentage (\%) } \\ 2001 & 1143 & 51 & 4.7 \\ 2002 & 1154 & 44 & 3.8 \\ 2003 & 1263 & 51 & 4.0 \\ 2004 & 1395 & 68 & 4.9 \\ 2005 & 1085 & 48 & 2.5 \\ 2006 & 1421 & 22 & 3.7 \\ 2007 & 1114 & 3 & 0.0008 \\ 2008 & 1212 & 5 & 0.004 \\ 2009 & 1143 & 3 & 0.003 \\ 2010 & 1209 & 7 & 0.006\end{array}$

Table II: Comparison of measles cases seen at the Wesley Guild hospital, Ilesa before and after nationwide supplemental measles immunization

$\begin{array}{llllll}\text { Cases } & \text { Before SIAs } & \text { After SIAs } & \text { Total } & \text { x2 } & \text { p-value } \\ & \mathbf{2 0 0 1 - 2 0 0 5} & \mathbf{2 0 0 6 - 2 0 1 0} & & & \\ \text { Measles cases } & 262 & 40 & 302 & 169.580 & 0.000 \\ \text { Non-measles cases } & 5778 & 6059 & 11837 & & \\ \text { TOTAL } & 6040 & 6099 & 12139 & & \end{array}$


Figure One: Measles cases admitted at the WGH, Ilesa year 2001 to 2010. Note - line shows when supplemental measles immunization was introduced in Nigeria.

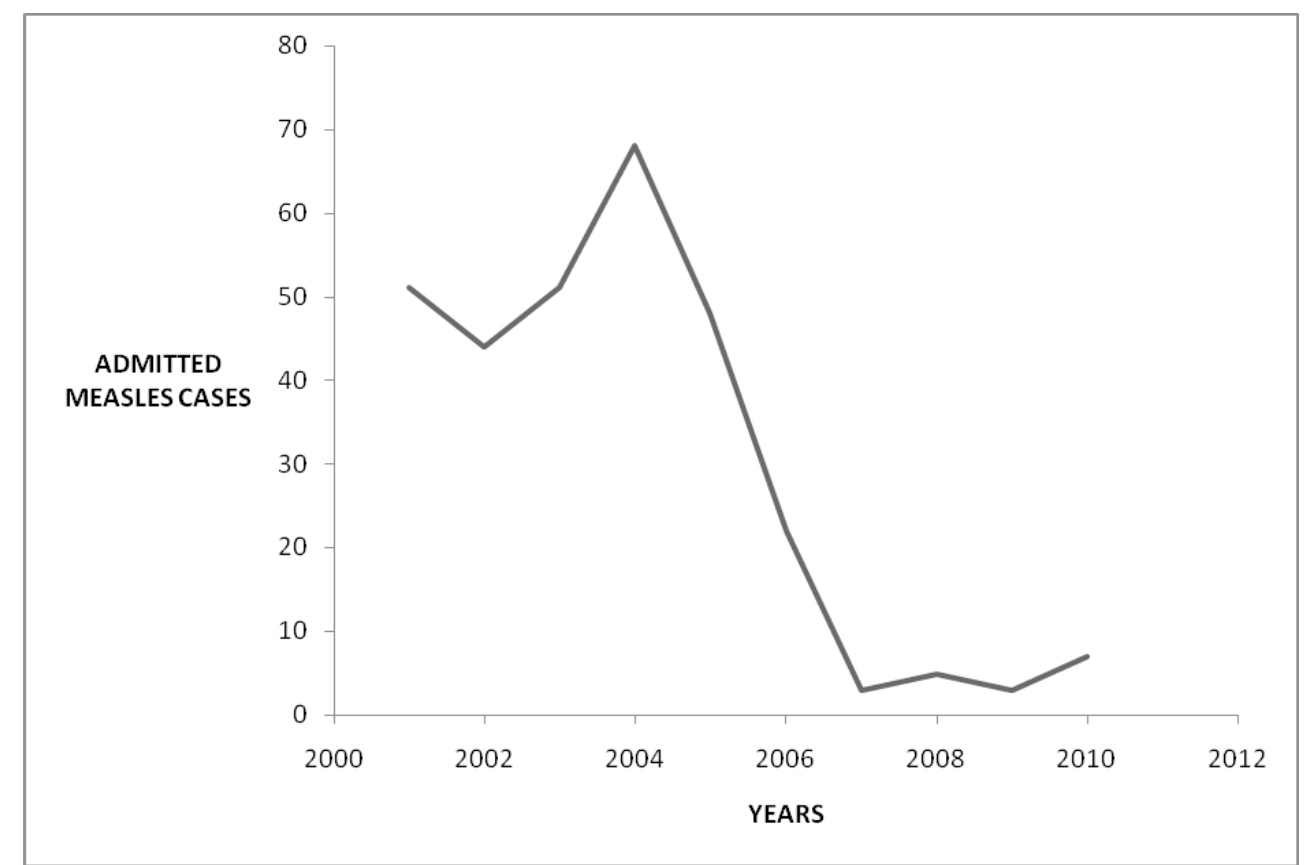

\section{Discussion}

Our study looked at the cases of complicated measles admitted into the Paediatric wards of the WGH, Ilesa, Nigeria over a ten year period which included a five year period after the introduction of supplemental measles immunization in the region. We noticed a significant reduction in the proportion of measles cases admitted in the periods after the introduction of the SIAs.

Routine immunization coverage rate in Nigeria and at the study location is low with measles immunization coverage ranging from $12.7 \%$ to $41.0 \%$ of children 12 23 months during the study period. ${ }^{5-7}$ Since no significant change in the routine immunization coverage rate and the total number of children admitted before and after the introduction of the supplementation measles immunization campaign as well as other childhood health interventions during the period, ${ }^{4-6}$ the remarkable reduction in the number of admitted measles cases observed in this study following SIAs can only be due to the supplemental measles immunization campaigns. This finding is in keeping with similar observations by Acharya $e$ al $^{10}$ in Latin America and Caribbean. SIAs have also been observed to be very effective in quickly reducing measles morbidity and mortality., 11-12

Children who were vaccinated have been reported to either be absolutely protected for life or in situation of vaccine failures from poor sero-conversion, problems with cold-chain maintenance and waning immunity to have milder infections. ${ }^{11}$ These milder forms may not require hospitalisation hence the remarkable reduction in the admitted cases of measles seen during the periods after the introduction of SIAs in our study. Though this study was limited by its retrospective nature with focus on only complicated cases and the fact that laboratory (serologic) confirmation of the admitted measles cases was not done, it still highlights the importance and efficacy of immunization in reducing morbidity due to vaccine-preventable disease like measles.

The impact of these SIAs can only be sustained only if they are a component of a comprehensive and longterm measles control strategy as described at the 63rd World Health Assembly in May 2010. ${ }^{13}$ Here delegates agreed to a global target of a $95 \%$ reduction in measles 
mortality by 2015 from the level seen in 2000, as well as to move towards eventual eradication. ${ }^{13}$ This should be based on four-prong actions of increasing routine immunization coverage, supplemental immunization campaigns, establishing case-based surveillance with laboratory confirmation and improved case management. ${ }^{13}$ If national governments do not have the ability to sustain implementation of a comprehensive strategy, SIAs may result only in a short-term reduction of measles deaths.

Continuous availability of the vaccines and the maintenance of the high immunization coverage momentum are needed to sustain the reduction in the cases of admitted measles cases. If this momentum is not sustained, reduction in herd immunity may gradually creep in as a result of immunization gaps making young infants with lower maternally acquired measles antibodies to become more susceptible. ${ }^{13}$ This backlash if not prevented can lead to devastating epidemic of measles like those experienced in Cuba and Honduras in the $16^{\text {th }}$ century where half to two-third of the susceptible children were killed by measles outbreaks. ${ }^{14}$

\section{Conclussion}

The apparent success towards elimination of Measles in Nigeria as evidenced by drastic fall in the cases of admitted children with complicated measles after the introduction of SIAs highlighted in our study need to be sustained by improving routine immunization coverage and maintain high herd immunity in the community. If these are not put in place, it will be like riding on a Tiger's back and we need to be careful not to end up in its belly.

\section{Reference}

1. Maldonado Y. Measles. In: Berman RE, Kliegman RM, Jenson HB (editors). Nelson textbook of Pediatrics 17th Edition Philadelphia Wb. Saunder 2004: 102630.

2. WHO guidelines on treating measles. http://www. who.int/vaccines-documents/ accessed 24th June, 2012.

3. World Health Organization, United Nations Children's Fund (2006) Measles mortality reduction and regional elimination strategic plan 2006-2010. Geneva, Switzerland: Available at: http://www.who.int/vaccines documents/Docs

4. World Health Organization (2006). Vaccine Preventable Disease Unit WHO.AFRO. www.gavialliance.org/ resources/GIN.

5. WHO Measles. Geneva. Weekly epidemiological record, No. 35, 28 august 2009.

6. Supplemental measles immunization in Nigeria. Available at: www.unicef.org/immunization/nigeria_30483. html. (accessed 24th June, 2012)

7. UNICEF.2011 State of the World's Children. New York: United Nations Children's Fund. www.unicef.org (accessed May 10, 2013)

8. Wharton M, Chorba TL, Vogt RL, Morse DL, Buehler JW. Case definitions for public health surveillance. MMWR Recomm. (1990) Rep 39: 1-43.

9. Oyedeji GA. Socioeconomic and cultural background of hospitalized children in Ilesa. Nig J. Paediatr. 1985; 13: 111-18.

10. Acharya A et al. Cost-effectiveness of measles elimination in Latin America and the

Caribbean: a prospective analysis. Vaccine, 2002, 20:3332-3341.

11. Akande T.M. A review of measles vaccine failure in developing countries. Nig med pract. 2007:52 (5); 112-6

12. Aaby P, Bukh J, Leerhoy J, Lisse I.M, Mordhorst C.H, Pederson I.R. Vaccinated children get milder measles infection: A community study from Guinea-Bissau. The J. Inf. Dis.1986; 154(5):858-63.

13."Sixty-third World Health Assembly Agenda provisional agenda item 11.15. Global eradication of measles". http://apps.who.int/gb/ebwha/pdf_files/ WHA63/A63_18-en.pdf. Retrieved 2 June 2010.

14. Byrne J.P Encyclopedia of Pestilence, Pandemics, and Plagues: A-M. ABC-CLIO. 2008; 413 - 4. Available from: http://books.google.com/books. updated June 2010 assessed 2nd July, 2012. 